\title{
CARBONO E NITROGÊNIO NAS FRAÇÕES GRANULOMÉTRICAS DA MATÉRIA ORGÂNICA DO SOLO, EM SISTEMAS DE CULTURAS SOB PLANTIO DIRETO( ${ }^{(1)}$
}

\author{
Bruna Raquel Winck ${ }^{(2)}$, Fabiane Machado Vezzani ${ }^{(3)}$, Jeferson Dieckow ${ }^{(3)}$, Nerilde \\ Favaretto $^{(3)}$ \& Rudimar Molin ${ }^{(4)}$
}

\section{RESUMO}

A qualidade do solo em plantio direto está relacionada ao sistema de culturas e pode ser avaliada pelo teor de matéria orgânica particulada ( $>53 \mu \mathrm{m})$, em razão da funcionalidade que essa fração proporciona ao solo e à sua sensibilidade às diferenças de manejo. Visando estudar a qualidade do solo em sistemas de culturas em plantio direto, este trabalho foi conduzido em experimento de longa duração (21 anos) em Latossolo Vermelho distrófico típico nos Campos Gerais do Paraná. Seis sistemas de culturas foram avaliados, em que trigo-TR (Triticum aestivum L.), soja-SO (Glycine max L.), milho-MI (Zea mays L.), aveia-preta-AV, para cobertura (Avena strigosa Schreb.), ervilhaca-ER, para cobertura (Vicia villosa Roth); azevém-AZ, para feno (Lolium multiflorum Lam.); ou alfafa-AL, para feno (Medicago sativa L.) compuseram os seguintes sistemas: TR-SO (referência), ER-MI-AV-SOTR-SO, ER-MI-TR-SO, AV-MI-TR-SO, AZ-MI-AZ-SO e AL-MI (milho a cada três anos). Os estoques de carbono orgânico total (COT), nitrogênio total (NT) e de C e N na matéria orgânica (MO) particulada $(>53 \mu \mathrm{m})$ e associada aos minerais $(<53 \mu \mathrm{m})$ foram determinados em 0-5, 5-10 e 10-20 cm. O sistema semiperene AL-MI apresentou os maiores estoques de COT e NT na camada de 0-20 cm $\left(63,6 \mathrm{Mg} \mathrm{ha}^{-1}\right.$ COT e 4,6 Mg ha-1 NT), com incrementos anuais de 0,23 $\mathrm{Mg} \mathrm{ha}^{-1} \mathrm{COT}$ e 0,03 $\mathrm{Mg} \mathrm{ha}^{-1}$ NT, em relação ao sistema TR-SO. O sistema AL-MI também teve os maiores estoques de $\mathrm{C}$ e $\mathrm{N}$ na $\mathrm{MO}$ particulada nessa camada (12,5 e $0,91 \mathrm{Mg} \mathrm{ha}^{-1}$, respectivamente), por causa da maior adição de fitomassa pelas raízes e a proteção física dos resíduos orgânicos. Os menores estoques de COT e NT na camada 0-20 cm ocorreram no sistema ER-MI-TR-SO (57,8 $\mathrm{Mg} \mathrm{ha}^{-1}$ COT e 4,03 $\mathrm{Mg} \mathrm{ha}^{-1} \mathrm{NT}$ ), sem apresentar incremento anual em relação ao sistema TR-SO. Os estoques de C e N na MO particulada foram de $10,4 \mathrm{e} 0,67 \mathrm{Mg} \mathrm{ha}^{-1}$, respectivamente. Essa tendência

(1) Parte da Dissertação de Mestrado da primeira autora apresentada ao Programa de Pós-Graduação em Ciência do Solo, Universidade Federal do Paraná - UFPR. Recebido para publicação em 4 de outubro de 2012 e aprovado em 30 de janeiro de 2014.

(2) Mestre em Ciência do Solo, Programa de Pós-Graduação em Ciência do Solo, UFPR. Rua dos Funcionários, 1540. CEP 80035050 Curitiba (PR). E-mail: bruna.winck@yahoo.com.br

(3) Professor Adjunto, Departamento de Solos e Engenharia Agrícola, UFPR. E-mail: vezzani@ufpr.br, jefersondieckow@ufpr.br (Bolsista Produtividade CNPq-1D), nfavaretto@ufpr.br (Bolsista Produtividade CNPq-2).

(4) Pesquisador da Fundação ABC - Pesquisa e Desenvolvimento Agropecuário. Rodovia PR 151, km 288. Caixa Postal 1003. CEP 84165-700 Castro (PR). E-mail: molin@fundacaoabc.org.br 
repetiu-se para as camadas individuais, com diferença significativa entre os sistemas na camada de 0-5 cm e não significativa, para as de 5-10 e 10-20 cm. Na média dos sistemas, a MO particulada contribuiu em torno de $30 \%$ para o estoque total de C na camada 0-5 cm. Rotação de culturas com espécies que tenham sistema radicular ativo por mais tempo, como o sistema semiperene AL-MI, tem potencial de incrementar o estoque total de $\mathrm{C}$ e N, especialmente da fração MO particulada, proporcionando funcionalidade ao solo e, consequentemente, qualidade.

Termos de indexação: rotação de culturas, adições de carbono e nitrogênio, matéria orgânica particulada, Latossolo.

\title{
SUMMARY: CARBON AND NITROGEN IN THE PARTICLE SIZE FRACTIONS OF SOIL ORGANIC MATTER IN CROP SYSTEMS UNDER NO- TILLAGE
}

\begin{abstract}
Soil quality in no-till systems is related to the crop systems and may be evaluated through particulate organic matter $(>53 \mu \mathrm{m})$ stock due to the functionality this property provides to the soil and its sensitivity to management practices. Aiming to assess soil quality no-till crop systems, this study was carried out through a long-term experiment (21 years) in a Latossolo Vermelho distrófico típico (typical Oxisol) in Ponta Grossa, PR, Brazil. Six crop systems were assessed. Wheat-TR (Triticum aestivum L.), soybean-SO (Glycine max L.), corn-MI (Zea mays $L$.), oat-AV (Avena strigosa Schreb.) as a cover crop, vetch-ER (Vicia villosa Roth) as a cover crop, ryegrass-AZ (Lolium multiflorum Lam) for hay, or alfalfa- $A L$ (Medicago sativa L.) for hay comprised the following systems: TR-SO (reference), ER-MI-AV-SO-TR-SO, ERMI-TR-SO, AV-MI-TR-SO, AZ-MI-AZ-SO and AL-MI (corn every three years). The stocks of total organic carbon (COT) and total $N(N T)$ and $C$ and $N$ in particulate $(>53 \mu \mathrm{m})$ and mineral-associated organic matter $(<53 \mu \mathrm{m})$ were determined at 0-5, $5-10$ and $10-20 \mathrm{~cm}$. The semiperennial AL-MI system had the greatest COT and NT stocks in the 0-20 cm layer (63.6 $M g ~ h a^{-1}$ COT and $4.6 \mathrm{Mg} \mathrm{ha} a^{-1} \mathrm{NT}$ ), and increased annual sequestration rates in relation to the TR-SO baseline system (0.23 Mg ha-1 year ${ }^{-1} \mathrm{COT}$ and $0.03 \mathrm{Mg} \mathrm{ha}^{-1}$ year $\left.{ }^{-1} \mathrm{NT}\right)$. The AL-MI system also had the highest $C$ and $N$ stocks in the particulate organic matter in the same layer (12.5 and $0.91 \mathrm{Mg} \mathrm{ha}^{-1}$, respectively) due to its higher Caddition from root plant matter and the physical protection of organic crop litter. The ER-MI-TR-SO system had the lowest $C$ and $N$ stocks in the $0-20 \mathrm{~cm}$ layer with values of totals stocks of 57.8 and $4.03 \mathrm{Mg} \mathrm{ha}^{-1}$, respectively, and without an increased annual sequestration rate because the stocks were similar to the TR$S O$ baseline system; $C$ and $N$ stocks in particulate organic matter were 10.4 and $0.67 \mathrm{Mg} \mathrm{ha}^{-1}$, respectively. This tendency was repeated for individual layers with a significant difference between systems in the $0-5 \mathrm{~cm}$ layer and no significant difference in the $5-10$ and $10-20 \mathrm{~cm}$ layers. The particulate organic matter contributed around $30 \%$ to total C stocks in the $0-5 \mathrm{~cm}$ layer on average in the systems. Crop rotations with species that have active root systems for a longer time, such as the semi-perennial AL-MI system, could increase total $C$ and $N$ stocks, especially the particulate organic matter, which promote soil functionality and, consequently, greater soil quality.
\end{abstract}

Index terms: crop rotation, carbon and nitrogen addition, particulate soil organic matter, Oxisol.

\section{INTRODUÇÃO}

A matéria orgânica (MO) é indicada como o atributo ideal para avaliar e monitorar a qualidade do solo de sistemas agrícolas (Bayer et al., 2001; Franzluebbers, 2002; Conceição et al., 2005; Shukla et al., 2006), por atuar em processos que melhoram as funções dele (Gregorich et al., 1994). Entre esses processos estão a diversidade e atividade biológica, a formação e estabilização de agregados, a infiltração e retenção de água, o aumento da CTC, o estoque e a ciclagem de nutrientes, a complexação de compostos e elementos prejudiciais aos ecossistemas (Six et al., 2002a,b;
Balota et al., 2004; Shukla et al., 2006; Vieira et al., 2009). Além disso, a MO atua no sequestro de C, contribuindo para a mitigação da emissão de gases de efeito estufa (Sisti et al., 2004; Diekow et al., 2005; Cerri et al., 2007; Zanatta et al., 2007).

A fração particulada da MO (C e N da fração $>53$ $\mu \mathrm{m})$, que consiste em fragmentos de plantas, animais e fungos, é sensível em detectar variações em práticas agrícolas e, por isso, é apontada como o melhor indicador para controlar alterações na qualidade do solo de sistemas de manejo (Bayer et al., 2001; 2004; Conceição et al., 2005; Diekow et al., 2005). A principal função da MO particulada é servir como fonte de 
energia e nutrientes aos organismos, o que desencadeia os processos de formação e estabilização dos agregados, transformações bioquímicas dos elementos e aumento de cargas no solo (Duxbury et al., 1989; Baldock \& Skjemstad, 2000; Six et al., 2002a). A entrada e a maior concentração de MO particulada ocorrem, principalmente, nos primeiros centímetros abaixo da superfície do solo, sendo diretamente influenciadas pela serapilheira e raízes (Duxbury et al., 1989). Dessa forma, o preparo do solo, a quantidade e qualidade dos resíduos culturais, a relação raiz/parte aérea e a frequência e duração dos cultivos interferem na dinâmica e no estoque de MO particulada (Janzen et al., 1998; Balesdent et al., 2000; Six et al., 2002a; Chevallier et al., 2004; Santos et al., 2011), e, portanto, nas propriedades do solo que conferem qualidade.

$\mathrm{O}$ sistema plantio direto (SPD) promove maior qualidade do solo em relação ao preparo convencional (Bayer et al., 2001; 2004; Diekow et al., 2005), em razão do não revolvimento do solo, da proteção física e manutenção da MO particulada e das propriedades emergentes que ela proporciona. Porém, a melhoria em qualidade do solo no SPD depende dos sistemas de culturas utilizados, pois o número e a alternância de espécies vegetais na rotação de culturas podem alterar a quantidade, qualidade e forma de adição de $\mathrm{C} \mathrm{e} \mathrm{N}$ ao solo (Oades, 1995; Santos et al., 2011). A disposição temporal das espécies, a utilização de culturas para cobertura e com elevada produção de fitomassa, tanto pela parte aérea como pelo sistema radicular (Janzen et al., 1998), também podem interferir diretamente os estoques de $\mathrm{C}$ e $\mathrm{N}$ nas diferentes frações da $\mathrm{MO}$ (Balesdent et al., 2000; Six et al., 2000; Bayer et al., 2001; Six et al., 2002a; Chevallier et al., 2004; Sisti et al., 2004; Diekow et al., 2005), o que tem potencial para modificar significativamente a qualidade do solo.

Considerando que a MO particulada expressa o potencial do solo em exercer suas funções e ter qualidade, o objetivo deste trabalho foi avaliar a qualidade do solo pelo comportamento das frações granulométricas da $\mathrm{MO}$ em sistemas de culturas sob plantio direto, com diferentes composições de espécies vegetais.

\section{MATERIAL E MÉTODOS}

A área experimental está localizada no município de Ponta Grossa, PR, nas coordenadas de $25^{\circ} 00^{\prime} 35^{\prime \prime}$ S e $50^{\circ} 09^{\prime} 16^{\prime \prime} \mathrm{W}$, numa altitude de $890 \mathrm{~m}$. O clima é subtropical úmido, mesotérmico, do tipo $\mathrm{Cfb}$ (Köppen). A temperatura média no mês mais quente fica entre 21 e $22{ }^{\circ} \mathrm{C}$; e no mais frio, entre 11 e $12{ }^{\circ} \mathrm{C}$. A precipitação pluvial média anual é de $1.554 \mathrm{~mm}$ e as geadas são frequentes no inverno (IAPAR, 2011).

O solo foi classificado como Latossolo Vermelho distrófico típico, A moderado (Embrapa, 2006), com declividade plana a suave ondulada. A textura é argilosa (449 $\mathrm{g} \mathrm{kg}^{-1}$ de areia, $101 \mathrm{~g} \mathrm{~kg}^{-1}$ de silte e $450 \mathrm{~g} \mathrm{~kg}^{-1}$ de argila) e os minerais predominantes na fração argila são, em ordem de predominância, caulinita e hematita.

O experimento foi instalado no inverno de $1989 \mathrm{e}$ compreende sistemas de rotações de culturas em plantio direto, com variação no número e na sequência temporal das espécies de plantas. $\mathrm{O}$ delineamento experimental foi blocos ao acaso, com quatro repetições e parcelas de $147 \mathrm{~m}^{2}(21 \times 7 \mathrm{~m})$. Seis sistemas de rotações de culturas foram avaliados e suas principais características são apresentadas no quadro 1. A sucessão anual TR-SO foi utilizada como referência. As principais características químicas do solo, determinadas após 21 anos de condução do experimento, estão apresentadas no quadro 2.

A amostragem de solo foi realizada em novembro de 2010, nas camadas de 0-5, 5-10 e 10-20 cm, em duas trincheiras por parcela, considerando as linhas e entrelinhas de cultivo. As camadas foram amostradas por escavação com espátula numa área delimitada por um gabarito de metal com dimensões internas de 40 $\times 25 \mathrm{~cm}$, inserido até $5 \mathrm{~cm}$ no solo. Chapas de metal foram utilizadas para delimitar exatamente a profundidade de 5,10 e $20 \mathrm{~cm}$ e para garantir o prumo das paredes e o nivelamento do fundo da trincheira. $\mathrm{O}$ solo retirado de cada camada foi pesado no campo; retirou-se uma subamostra para avaliação da umidade. Como o volume e a massa de solo de cada camada amostrada eram conhecidos, foi possível calcular a densidade do solo em cada camada, conforme o método de escavação (Blake \& Hartge, 1986).

Amostras de solo foram secas ao ar, moídas e passadas em peneira de $2,00 \mathrm{~mm}$. Uma alíquota foi moída até passar em malha de $0,50 \mathrm{~mm}$, e aproximadamente $20 \mathrm{mg}$ foram pesados para a determinação da concentração de carbono orgânico total (COT) e nitrogênio total (NT), pelo método de combustão seca, em analisador elementar (Vario EL III - Elementar). A quantificação dos estoques de COT e NT do solo levou em conta a massa equivalente de solo entre os tratamentos (Sisti et al., 2004) e, para tal, o sistema TR-SO foi a referência.

$\mathrm{O}$ fracionamento físico das amostras de solo para obtenção das frações granulométricas $>53$ e $<53 \mu \mathrm{m}$ seguiu o procedimento proposto por Cambardella \& Elliott (1992). Foram pesados $20 \mathrm{~g}$ de solo e adicionados $60 \mathrm{~mL}$ de hexametafosfato de sódio $\left(5 \mathrm{~g} \mathrm{~L}^{-1}\right)$, em frascos "snap-cap". A suspensão foi agitada durante $15 \mathrm{~h}$ em agitador horizontal e, posteriormente, passada em peneira de $53 \mu \mathrm{m}$. O material retido na peneira, constituído de matéria orgânica particulada e de areia, foi seco $\left(40^{\circ} \mathrm{C}\right)$, moído, pesado e analisado por combustão seca para a determinação de $\mathrm{CO}$ e N (Vario EL III - Elementar). As concentrações de C e N da fração $<53 \mu \mathrm{m}$ (matéria orgânica associada aos minerais) foram determinadas pela diferença entre as concentrações totais no solo e na fração $>53 \mu \mathrm{m}$. Também foram quantificados os estoques de $\mathrm{C}$ e $\mathrm{N}$ 
Quadro 1. Principais características dos sistemas de rotações de culturas manejados em plantio direto há 21 anos, em Latossolo Vermelho distrófico típico

\begin{tabular}{|c|c|c|c|c|c|c|c|}
\hline \multirow{2}{*}{ Sistema $^{(1)}$} & \multirow{2}{*}{ Ciclo } & \multirow{2}{*}{$\begin{array}{l}\text { Espécie/ } \\
\text { rotação }\end{array}$} & \multirow{2}{*}{$\begin{array}{c}\text { Resíduo } \\
\text { na } \text { coleta }^{(2)}\end{array}$} & \multirow[t]{2}{*}{ Relação G/L ${ }^{(3)}$} & \multicolumn{3}{|c|}{ Adição de $C^{(4)}$} \\
\hline & & & & & Parte aérea & Raíz & Total \\
\hline & & & & & -1 & $\mathrm{ha}^{-1} \mathrm{a}$ & \\
\hline TR-SO ${ }^{(5)}$ & Anual & 2 & Trigo & $1: 1$ & 3,78 & 1,91 & 5,69 \\
\hline ER-MI-AV-SO-TR-SO ${ }^{(6)}$ & Trianual & 6 & Aveia & $3: 3$ & 5,17 & 2,09 & 7,26 \\
\hline AV-MI-TR-SO ${ }^{(6)}$ & Bianual & 4 & Trigo & $3: 1$ & 6,20 & 2,36 & 8,56 \\
\hline ER-MI-TR-SO ${ }^{(6)}$ & Bianual & 4 & Trigo & $2: 2$ & 5,56 & 2,02 & 7,58 \\
\hline AZ-MI-AZ-SO ${ }^{(7)}$ & Bianual & 4 & Azevém & $3: 1$ & 5,33 & 3,11 & 8,44 \\
\hline AL-MI ${ }^{(8)}$ & Semiperene & 2 & Alfafa & $1: 1$ & 2,78 & 4,74 & 7,52 \\
\hline
\end{tabular}

(1) TR: trigo (Triticum aestivum L.); SO: soja (Glycine $\max$ (L.) Merr); ER: ervilhaca (Vicia villosa Roth); MI: milho (Zea mays L.); AV: aveia-preta (Avena strigosa Schreb.); AZ: azevém (Lolium multiflorum Lam.); e AL: alfafa (Medicago sativa L.). ${ }^{(2)}$ Referese ao resíduo cultural presente no momento da coleta de solo. ${ }^{(3)}$ Relação gramínea/leguminosa (número de espécies gramíneas/ número de espécies leguminosas). ${ }^{(4)}$ Compilado de Santos et al. (2011). ${ }^{(5)}$ Sistema referência, exclusivo para produção de grãos. (6) A ervilhaca e, ou, aveia-preta são utilizadas como plantas de cobertura de inverno, manejadas com dessecação. ${ }^{\left({ }^{7}\right)} \mathrm{O}$ azevém é utilizado para a produção de feno (média de três cortes por ciclo) e dessecado antes da semeadura da cultura de verão. ${ }^{(8)}$ A alfafa é cultivada para a produção de feno (média de sete a oito cortes anuais) e a cada três anos há um cultivo de milho.

Quadro 2. Principais características químicas da camada de 0-20 cm de um Latossolo Vermelho distrófico típico sob sistemas de rotações de culturas em plantio direto há 21 anos

\begin{tabular}{|c|c|c|c|c|c|c|c|c|c|c|c|}
\hline \multirow{2}{*}{ Sistema $^{(1)}$} & \multicolumn{2}{|c|}{$\mathbf{p H}$} & \multirow{2}{*}{$\mathrm{Al}$} & \multirow{2}{*}{$\mathrm{H}+\mathrm{Al}$} & \multirow{2}{*}{$\mathbf{C a}$} & \multirow{2}{*}{ Mg } & \multirow{2}{*}{$\mathbf{K}$} & \multirow{2}{*}{ SB } & \multirow{2}{*}{ CTC } & \multirow{2}{*}{ V } & \multirow{2}{*}{$\mathbf{P}$} \\
\hline & $\mathrm{CaCl}_{2}$ & SMP & & & & & & & & & \\
\hline & & & & & & $\operatorname{nol}_{c} d$ & & & & $\%$ & $\mathrm{mg} \mathrm{dm}{ }^{-3}$ \\
\hline TR-SO & 5,4 & 6,0 & 0,0 & 5,0 & 5,0 & 2,0 & 0,5 & 7,5 & 12,5 & 60,0 & 27,2 \\
\hline ER-MI-AV-SO-TR-SO & 5,4 & 6,0 & 0,0 & 5,0 & 3,8 & 1,6 & 0,6 & 6,0 & 11,0 & 54,3 & 18,7 \\
\hline ER-MI-TR-SO & 5,2 & 6,0 & 0,0 & 5,0 & 4,6 & 2,0 & 0,5 & 7,1 & 12,1 & 58,8 & 29,8 \\
\hline AV-MI-TR-SO & 5,4 & 6,1 & 0,0 & 7,8 & 4,5 & 1,9 & 0,5 & 6,9 & 14,7 & 46,9 & 17,3 \\
\hline AZ-MI-AZ-SO & 5,0 & 5,8 & 0,0 & 5,8 & 3,9 & 1,8 & 0,3 & 6,0 & 11,8 & 50,9 & 30,7 \\
\hline AL-MI & 4,9 & 5,8 & 0,0 & 5,8 & 4,2 & 1,0 & 0,7 & 5,9 & 11,7 & 50,4 & 79,0 \\
\hline
\end{tabular}

(1) TR: trigo (Triticum aestivum L.); SO: soja (Glycine max (L.) Merr); ER: ervilhaca (Vicia villosa Roth); MI: milho (Zea mays L.); AV: aveia-preta (Avena strigosa Schreb.); AZ: azevém (Lolium multiflorum Lam.); e AL: alfafa (Medicago sativa L.).

nas duas frações granulométricas, levando em conta todas as correções de unidades e de massa equivalente.

Os dados foram submetidos à análise de variância (ANOVA) e as médias comparadas pelo teste de Tukey a $5 \%$. A variável "camada" foi analisada como subparcela. Foi realizada análise de regressão entre os estoques de COT e NT do solo e das frações granulométricas $>53$ e $<53 \mu \mathrm{m}$. As análises foram realizadas com o programa ASSISTAT.

\section{RESULTADOS E DISCUSSÃO}

O número e a sequência de espécies vegetais na rotação de culturas em plantio direto influenciou significativamente o estoque de NT, C e N da MO particulada, na camada de $0.5 \mathrm{~cm}$; e o estoque acumulado de COT, NT e N da MO, na camada de
0-20 cm (Quadro 3). No entanto, o padrão observado na camada de $0-20 \mathrm{~cm}$, tanto no estoque de $\mathrm{C}$ e $\mathrm{N}$ total como nas frações da MOS, provavelmente foi influenciado pela camada superficial do solo. Isso acontece porque em sistemas de rotação de culturas em plantio direto a inserção de material orgânico ao solo ocorre na superfície, pela decomposição da serapilheira proveniente da parte aérea e do sistema radicular das culturas, principalmente gramíneas.

$\mathrm{Na}$ camada de $0-5 \mathrm{~cm}$, somente o $\mathrm{N}$ variou quanto ao estoque total; os maiores estoques de NT ocorreram no sistema AL-MI (1,64 $\left.\mathrm{Mg} \mathrm{ha}^{-1} \mathrm{NT}\right)$ e ER-MI-AV-SOTR-SO (1,52 Mg ha-1 NT). Na camada de 0-20 cm, o estoque de COT no sistema AL-MI $\left(63,6 \mathrm{Mg}^{-1}{ }^{-1} \mathrm{COT}\right.$ e $4,60 \mathrm{Mg} \mathrm{ha}^{-1} \mathrm{NT}$ ) diferiu somente dos sistemas ER-MITR-SO (57,8 Mg ha-1 COT e 4,03 $\mathrm{Mg} \mathrm{ha}^{-1} \mathrm{NT}$ ); e o estoque de NT, dos sistemas ER-MI-TR-SO e TR-SO (4,03 e $3,98 \mathrm{Mg} \mathrm{ha}^{-1} \mathrm{NT}$, respectivamente). Porém, o sistema AL-MI não distinguiu dos demais sistemas de culturas. 
O C da MO particulada variou entre os sistemas de culturas na camada de $0-5 \mathrm{~cm}$; e o $\mathrm{N}$ da $\mathrm{MO}$ particulada, nas camadas 0-5 e 0-20 cm. Assim como no COT e NT, os maiores estoques ocorreram novamente no sistema AL-MI, em comparação ao TRSO e ER-MI-TR-SO. Essa tendência se repetiu para as camadas de 5-10 e 10-20 cm, porém com diferença não significativa entre os sistemas (Quadro 3).

Certamente, os estoques de $\mathrm{C}$ e $\mathrm{N}$ na matéria orgânica total e na fração particulada no sistema AL-
MI, nas camadas de 0-5 e 0-20 cm, foram influenciados pelo cultivo de alfafa por 2,5 anos na rotação. A alfafa é uma leguminosa semiperene fixadora de $\mathrm{N}$ atmosférico, que apresenta alta adição de $\mathrm{C}$ pelo sistema radicular (Santos et al., 2011) e incorpora de 0,12 a 0,25 Mg ha-1 ano $^{-1}$ NT (Freire, 1992; Zhu et al., 1998), o que pode ter resultado no incremento de N observado no solo (Quadro 3). Em um estudo neste mesmo experimento, em 2006, Santos et al. (2011) estimaram uma adição de $7,52 \mathrm{Mg} \mathrm{ha}^{-1} \mathrm{ano}^{-1} \mathrm{C}$ pelo

Quadro 3. Estoques de C orgânico total (COT) e N total (NT) do solo e de C e N das frações granulométricas da matéria orgânica (MO) particulada $(>53 \mu \mathrm{m})$ e $M O$ associada aos minerais $(<53 \mu \mathrm{m})$, na camada acumulada de 0-20 cm e nas camadas de 0-5, 5-10, 10-20 cm de um Latossolo Vermelho distrófico típico, sob sistemas de rotações de culturas em plantio direto há 21 anos

\begin{tabular}{|c|c|c|c|c|c|c|}
\hline \multirow{2}{*}{ Sistema $^{(1)}$} & \multicolumn{2}{|c|}{ Total } & \multicolumn{2}{|c|}{ MO particulada } & \multicolumn{2}{|c|}{ MO associada aos minerais } \\
\hline & COT & NT & C & $\mathbf{N}$ & C & $\mathbf{N}$ \\
\hline & \multicolumn{6}{|c|}{$-\mathrm{Mg} \mathrm{ha}^{-1}$} \\
\hline & \multicolumn{6}{|c|}{$0-5 \mathrm{~cm}$} \\
\hline TR-SO & $18,0 \mathrm{aB}^{(2)}$ & $1,33 \mathrm{bB}$ & $5,6 \mathrm{bA}$ & $0,41 \mathrm{bA}$ & $12,5^{\text {ns }} B$ & $0,92^{\text {ns }} B$ \\
\hline ER-MI-AV-SO-TR-SO & $18,5 \mathrm{aB}$ & $1,52 \mathrm{abA}$ & $6,6 \mathrm{abA}$ & $0,47 \mathrm{abA}$ & $11,8 \mathrm{~B}$ & $1,05 \mathrm{~B}$ \\
\hline ER-MI-TR-SO & $17,4 \mathrm{aB}$ & $1,35 \mathrm{bB}$ & 5,7 bA & $0,40 \mathrm{bA}$ & $11,8 \mathrm{~B}$ & $0,95 \mathrm{~B}$ \\
\hline AV-MI-TR-SO & $18,5 \mathrm{aB}$ & $1,42 \mathrm{bB}$ & $6,6 \mathrm{abA}$ & $0,45 \mathrm{abA}$ & $11,9 \mathrm{~B}$ & $0,97 \mathrm{~B}$ \\
\hline AZ-MI-AZ-SO & $18,6 \mathrm{aB}$ & $1,42 \mathrm{bB}$ & $6,4 \mathrm{abA}$ & $0,44 \mathrm{abA}$ & $12,3 \mathrm{~B}$ & $0,98 \mathrm{~B}$ \\
\hline \multirow[t]{2}{*}{ AL-MI } & $20,0 \mathrm{aB}$ & $1,64 \mathrm{aB}$ & 6,8 aA & 0,52 aA & $13,2 \mathrm{~B}$ & $1,12 \mathrm{~B}$ \\
\hline & \multicolumn{6}{|c|}{$5-10 \mathrm{~cm}$} \\
\hline TR-SO & $14,4^{\mathrm{ns}} \mathrm{C}$ & $1,04^{\mathrm{ns}} \mathrm{C}$ & $2,5^{\mathrm{ns}} \mathrm{B}$ & $0,16^{\text {ns }} B$ & $11,9^{\text {ns }} B$ & $0,88^{\text {ns }} B$ \\
\hline ER-MI-AV-SO-TR-SO & $14,7 \mathrm{C}$ & $1,09 \mathrm{~B}$ & $2,6 \mathrm{~B}$ & $0,18 \mathrm{~B}$ & $12,1 \mathrm{~B}$ & $0,91 \mathrm{C}$ \\
\hline ER-MI-TR-SO & $14,4 \mathrm{C}$ & $1,01 \mathrm{C}$ & $2,3 \mathrm{~B}$ & $0,14 \mathrm{~B}$ & $12,1 \mathrm{~B}$ & $0,87 \mathrm{~B}$ \\
\hline AV-MI-TR-SO & $14,7 \mathrm{C}$ & $1,02 \mathrm{C}$ & $2,5 \mathrm{~B}$ & $0,15 \mathrm{~B}$ & $12,2 \mathrm{~B}$ & $0,87 \mathrm{~B}$ \\
\hline AZ-MI-AZ-SO & $15,6 \mathrm{C}$ & $1,09 \mathrm{C}$ & $1,7 \mathrm{C}$ & $0,11 \mathrm{~B}$ & $14,0 \mathrm{~B}$ & $0,98 \mathrm{~B}$ \\
\hline \multirow[t]{2}{*}{ AL-MI } & $15,5 \mathrm{C}$ & $1,16 \mathrm{C}$ & $2,4 \mathrm{~B}$ & $0,17 \mathrm{~B}$ & $13,0 \mathrm{~B}$ & $0,99 \mathrm{~B}$ \\
\hline & \multicolumn{6}{|c|}{$10-20 \mathrm{~cm}$} \\
\hline TR-SO & $26,4^{\mathrm{ns}} \mathrm{A}$ & $1,61^{\mathrm{ns}} \mathrm{A}$ & $2,5^{\mathrm{ns}} \mathrm{B}$ & $0,12^{\text {ns }} B$ & $23,9^{\text {ns }} A$ & $1,48^{\text {ns }} A$ \\
\hline ER-MI-AV-SO-TR-SO & $26,0 \mathrm{~A}$ & $1,61 \mathrm{~A}$ & $2,4 \mathrm{~B}$ & $0,14 \mathrm{~B}$ & $23,5 \mathrm{~A}$ & $1,47 \mathrm{~A}$ \\
\hline ER-MI-TR-SO & $26,0 \mathrm{~A}$ & $1,67 \mathrm{~A}$ & $2,5 \mathrm{~B}$ & $0,14 \mathrm{~B}$ & $23,5 \mathrm{~A}$ & $1,53 \mathrm{~A}$ \\
\hline AV-MI-TR-SO & $25,4 \mathrm{~A}$ & $1,65 \mathrm{~A}$ & $2,7 \mathrm{~B}$ & $0,15 \mathrm{~B}$ & $22,7 \mathrm{~A}$ & $1,50 \mathrm{~A}$ \\
\hline AZ-MI-AZ-SO & $26,9 \mathrm{~A}$ & $1,57 \mathrm{~A}$ & $2,6 \mathrm{~B}$ & $0,15 \mathrm{~B}$ & $24,3 \mathrm{~A}$ & $1,42 \mathrm{~A}$ \\
\hline AL-MI & $28,1 \mathrm{~A}$ & $1,80 \mathrm{~A}$ & $3,2 \mathrm{~B}$ & $0,22 \mathrm{~B}$ & $24,9 \mathrm{~A}$ & $1,58 \mathrm{~A}$ \\
\hline CV (\%) (sistema) & 7,2 & 10,0 & 15,4 & 21,0 & 8,6 & 12,0 \\
\hline \multirow[t]{2}{*}{ CV (\%) (camada) } & 5,5 & 5,4 & 13,6 & 14,5 & 6,5 & 7,0 \\
\hline & \multicolumn{6}{|c|}{$0-20 \mathrm{~cm}$} \\
\hline TR-SO & $58,8 \mathrm{ab}$ & $3,98 \mathrm{~b}$ & $10,6^{\mathrm{ns}}$ & $0,69 \mathrm{~b}$ & $48,2^{\text {ns }}$ & $3,28^{\text {ns }}$ \\
\hline ER-MI-AV-SO-TR-SO & $59,1 \mathrm{ab}$ & $4,22 \mathrm{ab}$ & 11,7 & $0,79 \mathrm{ab}$ & 47,5 & 3,43 \\
\hline ER-MI-TR-SO & $57,8 \mathrm{~b}$ & $4,03 \mathrm{~b}$ & 10,4 & $0,67 \mathrm{~b}$ & 47,4 & 3,36 \\
\hline AV-MI-TR-SO & $58,7 \mathrm{ab}$ & $4,09 \mathrm{ab}$ & 11,8 & $0,75 \mathrm{ab}$ & 46,9 & 3,33 \\
\hline AZ-MI-AZ-SO & $61,2 \mathrm{ab}$ & $4,08 \mathrm{ab}$ & 10,7 & $0,70 \mathrm{ab}$ & 50,5 & 3,39 \\
\hline AL-MI & $63,6 \mathrm{a}$ & $4,60 \mathrm{a}$ & 12,5 & $0,91 \mathrm{a}$ & 51,1 & 3,69 \\
\hline CV (\%) (sistema) & 4,1 & 5,8 & 8,9 & 12,1 & 5,0 & 6,9 \\
\hline
\end{tabular}

(1) TR: trigo (Triticum aestivum L.); SO: soja (Glycine max (L.) Merr); ER: ervilhaca (Vicia villosa Roth); MI: milho (Zea mays L.); AV: aveia-preta (Avena strigosa Schreb.); AZ: azevém (Lolium multiflorum Lam.); AL: alfafa (Medicago sativa L.). ${ }^{(2)}$ Letras minúsculas comparam médias entre sistemas de rotação, numa mesma camada; e letras maiúsculas comparam médias entre camadas, num mesmo sistema de rotação. Teste Tukey a $5 \%$. 
sistema AL-MI, sendo $63 \%$ dessa adição $\left(4,74 \mathrm{Mg}\right.$ ha $^{-1}$ $\mathrm{ano}^{-1} \mathrm{C}$ ) proveniente das raízes; enquanto no sistema TR-SO a adição foi estimada em $5,69 \mathrm{Mg} \mathrm{ha}^{-1}$ ano $^{-1} \mathrm{C}$, sendo apenas $34 \%$ oriundo de raízes, o que explica os baixos estoques de $\mathrm{C}$ e $\mathrm{N}$ totais nas frações granulométricas da MO neste sistema (Quadro 3).

Raízes de alfafa constituem-se numa importante fonte de MO particulada, que é protegida fisicamente no solo (Balesdent \& Balabane, 1996; Rasse et al., 2005). Ao atuarem na formação e estabilização de macroagregados, as raízes em troca recebem a proteção física, reduzindo assim sua taxa de mineralização (Balesdent \& Balabane, 1996; Rasse et al., 2005). Além disso, a proteção física da MO particulada é mais eficiente em sistemas cujo tempo de permanência da cultura no campo é maior e que adicionam mais resíduos orgânicos pelas raízes (Janzen et al., 1998), como ocorre no sistema com alfafa.

Os baixos estoques de $\mathrm{C}$ e $\mathrm{N}$ no sistema bianual ER-MI-TR-SO (Quadro 3) observados nas camadas de 0-5 e 0-20 cm podem estar relacionados com a sequência das culturas na rotação. A sequência das culturas possivelmente promoveu o "efeito priming" (Kuzyakov et al., 2000; Kuzyakov, 2002; Kalbitz \& Kaiser, 2008), cujo processo se refere ao aumento da decomposição da MO do solo pela elevação da disponibilidade de N, prontamente disponível, oriunda de resíduos orgânicos de leguminosas, como a soja e ervilhaca em safras seguidas nesse sistema. Dessa forma, mesmo com adição total de $\mathrm{C}$ superior em relação ao sistema referência TR-SO (Quadro 1), a variação entre os estoques total de $\mathrm{C}$ de ambos os sistemas não ultrapassou $4 \%$ na camada de $0-5 \mathrm{~cm}$ e $2 \%$, na de 0-20 cm. Além disso, de acordo com Santos et al. (2011), o sistema ER-MI-TR-SO tem baixa adição de resíduos pelo sistema radicular $\left(2,02 \mathrm{Mg} \mathrm{ha}^{-1} \mathrm{ano}^{-1} \mathrm{C}\right)$ em relação à adição total $\left(7,58 \mathrm{Mg} \mathrm{ha}^{-1} \mathrm{ano}^{-1} \mathrm{C}\right)$, o que pode ter contribuído para o baixo estoque de $\mathrm{C} \mathrm{e} \mathrm{N}$, refletindo em maiores variações estatísticas na fração particulada (Quadro 3).

Os sistemas trianual ER-MI-AV-SO-TR-SO e bianuais AV-MI-TR-SO e AZ-MI-AZ-SO não diferiram do sistema AL-MI e dos sistemas ER-MI-TR-SO e TR$\mathrm{SO}$, apresentando valores intermediários para o estoque de NT, C e N $>53 \mu \mathrm{m}$, na camada de $0-5 \mathrm{~cm}$, e para COT, NT e N $>53 \mu \mathrm{m}$, na de $0-20 \mathrm{~cm}$ (Quadro 3). O sistema trianual ER-MI-AV-SO-TR-SO utiliza a rotação gramínea com elevada produção de fitomassa e com alta relação $\mathrm{C} / \mathrm{N}$ (milho, aveia e trigo), associada à utilização de leguminosas (ervilhaca e soja), numa relação gramínea/leguminosa (G/L) de 3:3. A composição vegetal e a sequência das culturas pode influenciar positivamente a produção de fitomassa e, por sua vez, o estoque de $\mathrm{C}$ e $\mathrm{N}$ ao longo do tempo, como já reportado por Lovato et al.(2004) e Weber \& Mielniczuk (2009). Porém, Santos et al. (2011) observaram que o sistema trianual deste estudo apresentou menor adição de resíduos $\left(7,26 \mathrm{Mg} \mathrm{ha}^{-1} \mathrm{ano}^{-1}\right)$ em relação ao AV-MI-TR-SO (8,56 $\left.\mathrm{Mg} \mathrm{ha}^{-1} \mathrm{ano}^{-1}\right)$ e AZ-MI-AZ-SO $\left(8,44 \mathrm{Mg} \mathrm{ha}^{-1}\right.$ ano $\left.^{-1}\right)$. Isso possivelmente está associado ao fato de o sistema trianual possuir um cultivo de milho, com alta adição de resíduos, para dois de soja, com baixa adição, o que reduz a adição média anual em relação àquela dos sistemas bianuais, que possuem uma safra de milho para uma de soja. Mesmo com adições e características distintas, esses três sistemas tiveram os estoques de $\mathrm{C}$ e $\mathrm{N}$ totais e a $\mathrm{MO}$ particulada semelhantes. Os sistemas bianuais têm relação G/L de 3:1, ou seja, maior proporção de espécies com alto aporte de fitomassa e de alta relação $\mathrm{C} / \mathrm{N}$, o que resulta em alta adição de C e N (Quadro 1) no sistema e menor decomposição desses. No trianual ER-MI-AV-SO-TR$\mathrm{SO}$, mesmo com mais baixa adição, provavelmente as características formadas de diversidade e estrutura de solo estão promovendo maior estabilização do C adicionado (Vezzani \& Mielniczuk, 2011).

As diferenças nos estoques de $\mathrm{C}$ e $\mathrm{N}$ na $\mathrm{MO}$ particulada entre os sistemas de culturas nas camadas de $0-5 \mathrm{~cm}$ (Quadro 3) refletem as características das plantas utilizadas e a sequência dessas no tempo. Além disso, a variação nos estoques da fração particulada na camada superficial do solo influencia o estoque acumulado na camada de $0-20 \mathrm{~cm}$, o que refletiu em diferença significativa no estoque de $\mathrm{N}$ da MO particulada (Six et al., 2002a; Conceição et al., 2005). Essa variação observada confirma a sensibilidade desse compartimento em diferenciar sistemas de culturas, como constataram vários autores (Bayer et al., 2001; Diekow et al., 2005; Ashagrie et al. 2007; Santos et al., 2011).

A ausência de diferença nos estoques de $\mathrm{C}$ e $\mathrm{N}$ da MO associada aos minerais entre os sistemas (Quadro 3) demonstra a menor sensibilidade dessa fração à variação no número e na sequência de espécies vegetais e na rotação de culturas em sistemas de longa duração. Este fato se explica em razão de os estoques nessa fração serem fortemente dependentes dos teores de argila e das características mineralógicas dessa (Baldock \& Skjemstad, 2000; Six et al., 2004), que atuam nas interações com os compostos orgânicos mais decompostos, diminuindo sua variabilidade no solo pelos diferentes sistemas de culturas ao longo do tempo. Porém, é importante ressaltar que houve tendência a maiores estoques de $\mathrm{C} \mathrm{e} \mathrm{N}$ da $\mathrm{MO}$ associada aos minerais no sistema AL-MI. Essa tendência devese à maior adição de resíduos orgânicos pela raiz (Quadro 1), cuja estabilização desses compostos orgânicos pode estar seguindo os mecanismos para $\mathrm{C}$ rizodepositado, apresentados por Rasse et al. (2005), proporcionando ligeiro aumento em relação aos demais sistemas.

\section{Proporções de C e N das frações granulométricas no estoque total do solo}

As proporções de $\mathrm{C}$ e $\mathrm{N}$ da $\mathrm{MO}$ particulada no estoque total ficaram em torno de 31 a $36 \%$ para C e 29 a $32 \%$ para N, na camada superficial (Figura 1). As proporções diminuíram em profundidade, porque esse compartimento é influenciado pela adição recente 
de resíduos, que, no caso do sistema plantio direto, os resíduos da parte aérea permanecem na superfície e não são incorporados ao solo. Com o avanço da decomposição e mineralização da $\mathrm{MO}$, a fração particulada tende a um decréscimo na superfície com aumento relativo da $\mathrm{MO}$ associada aos minerais em profundidade. Assim, somente com o aporte contínuo de resíduos orgânicos pelos sistemas de culturas é que há a manutenção e as maiores proporções dessa fração no solo (Baldock \& Skjemstad, 2000; Balesdent et al.,
2000; Six et al., 2002a; 2004). As raízes também podem ter interferido nessa variação na proporção de MO particulada no solo entre as camadas, principalmente em sistemas com gramíneas, uma vez que há grande volume de raízes que permanecem nas primeiras camadas do solo (Balesdent \& Balabane, 1996; Rasse et al., 2005).

As maiores proporções no estoque total ocorreram na $\mathrm{MO}$ associada aos minerais, com valores entre 64 e 69 \% para C e 68 a $71 \%$ para N, na camada de

$0-5 \mathrm{~cm}$

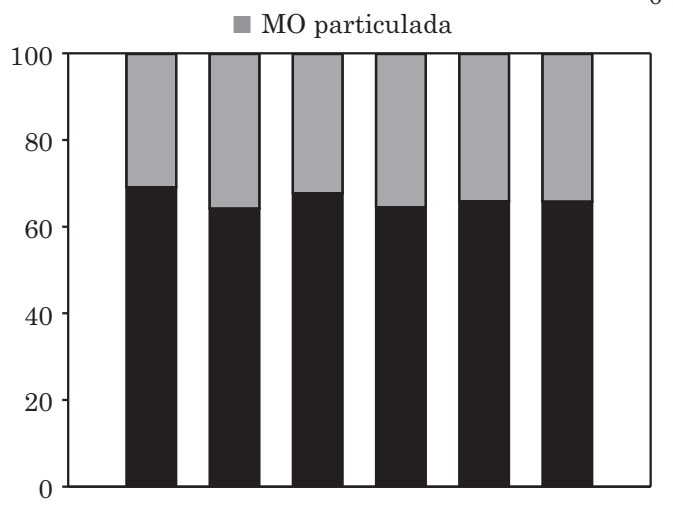

- MO associada aos minerais

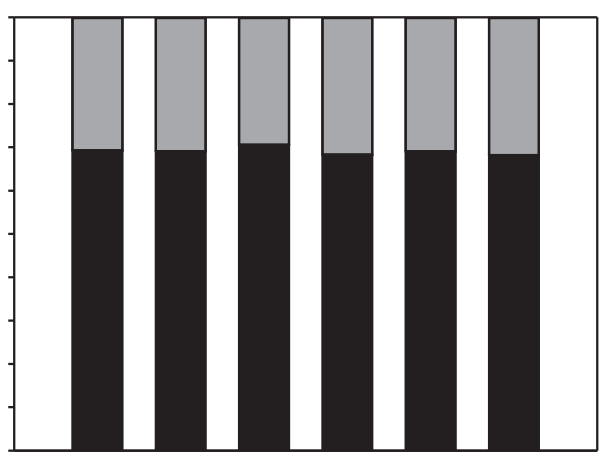

$5-10 \mathrm{~cm}$
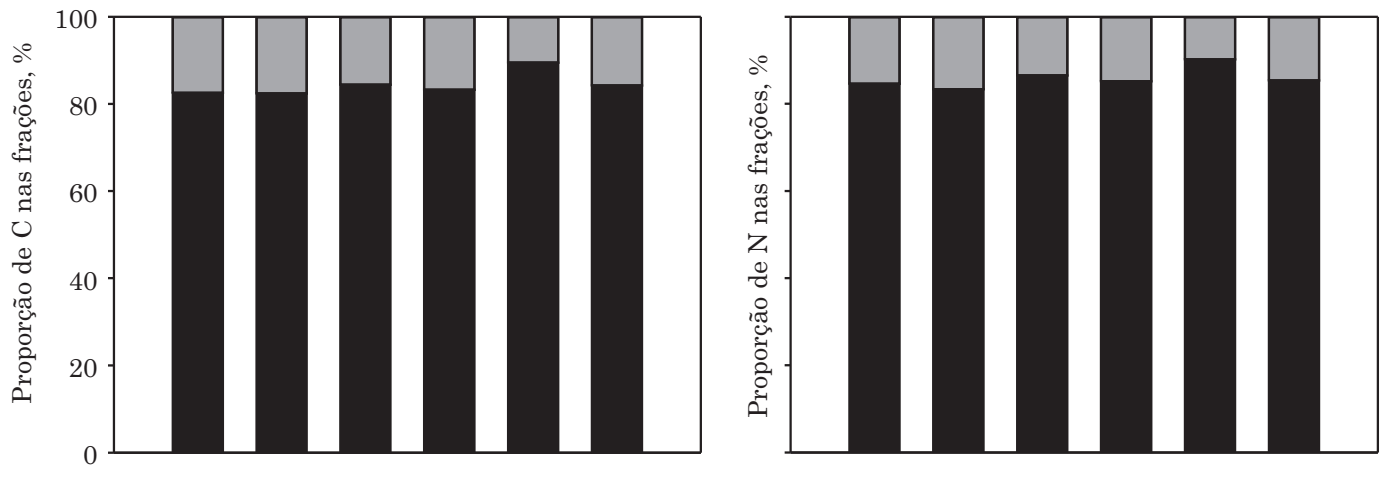

$10-20 \mathrm{~cm}$
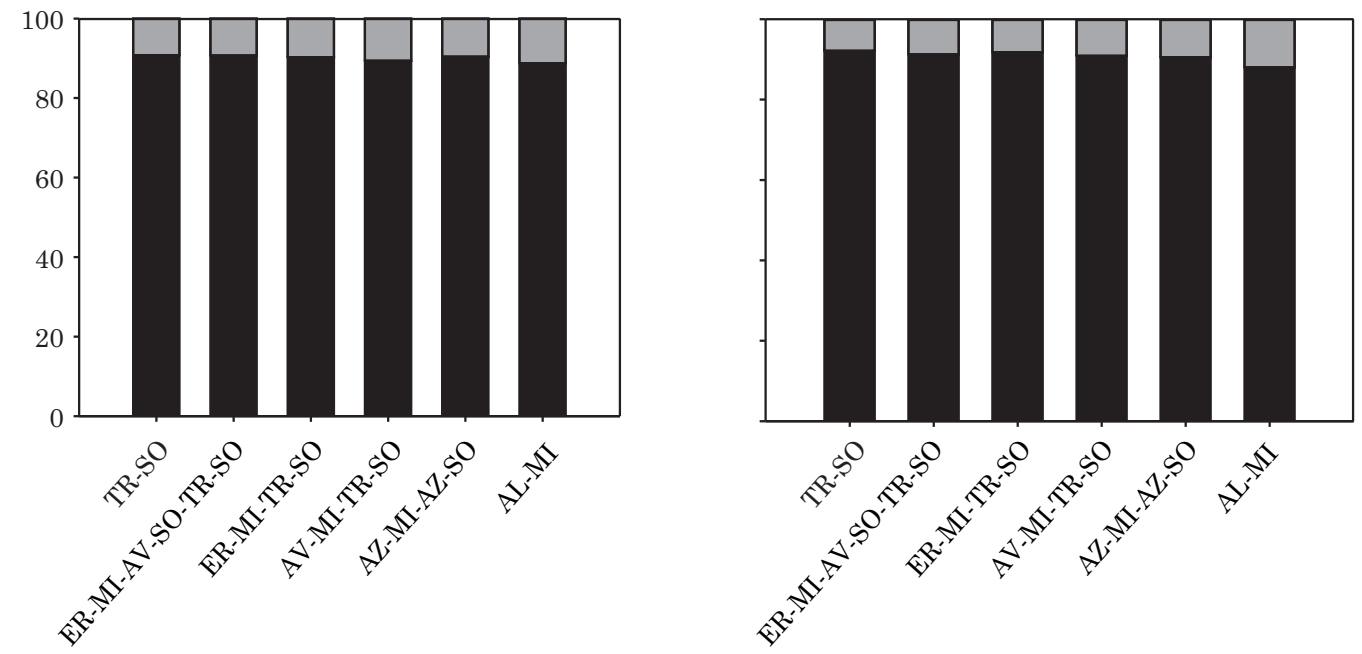

Figura 1. Proporções de $\mathrm{C}$ e $\mathrm{N}$ das frações granulométricas da matéria orgânica (MO) particulada (>53 $\mu \mathrm{m})$ e MO associada aos minerais $(<53 \mu \mathrm{m})$ no estoque total de $\mathrm{C}$ e $\mathrm{N}$ em sistemas de culturas em rotação sob plantio direto há 21 anos, em Latossolo Vermelho distrófico típico. 
0-5 cm (Figura 1). Isso porque as frações granulométricas mais finas desse solo, sobretudo a argila, apresentam minerais com maior capacidade de interação com a MO. Em profundidade, as proporções de $\mathrm{C}$ e $\mathrm{N}$ na $\mathrm{MO}$ associada aos minerais tenderam a aumentar, com valores próximos a $90 \%$ (Figura 1). Isso se justifica pela menor contribuição de MO particulada oriunda de serapilheira e raízes superficiais de gramíneas. Valores próximos às proporções encontradas neste estudo para sistemas de plantio direto foram reportados por Bayer et al. (2004), Conceição et al. (2005) e Diekow et al. (2005).

\section{Relações entre estoques de $\mathrm{C}$ e $\mathrm{N}$ totais e das frações granulométricas}

As regressões entre os estoques de $\mathrm{C}$ e $\mathrm{N}$ totais e as frações granulométricas da $\mathrm{MO}$ em todas as camadas apresentaram comportamento linear (Figura 2). Diekow et al. (2005) afirmaram que na fração argila a interação da MO com os minerais apresenta um limite máximo de saturação, tendendo a uma
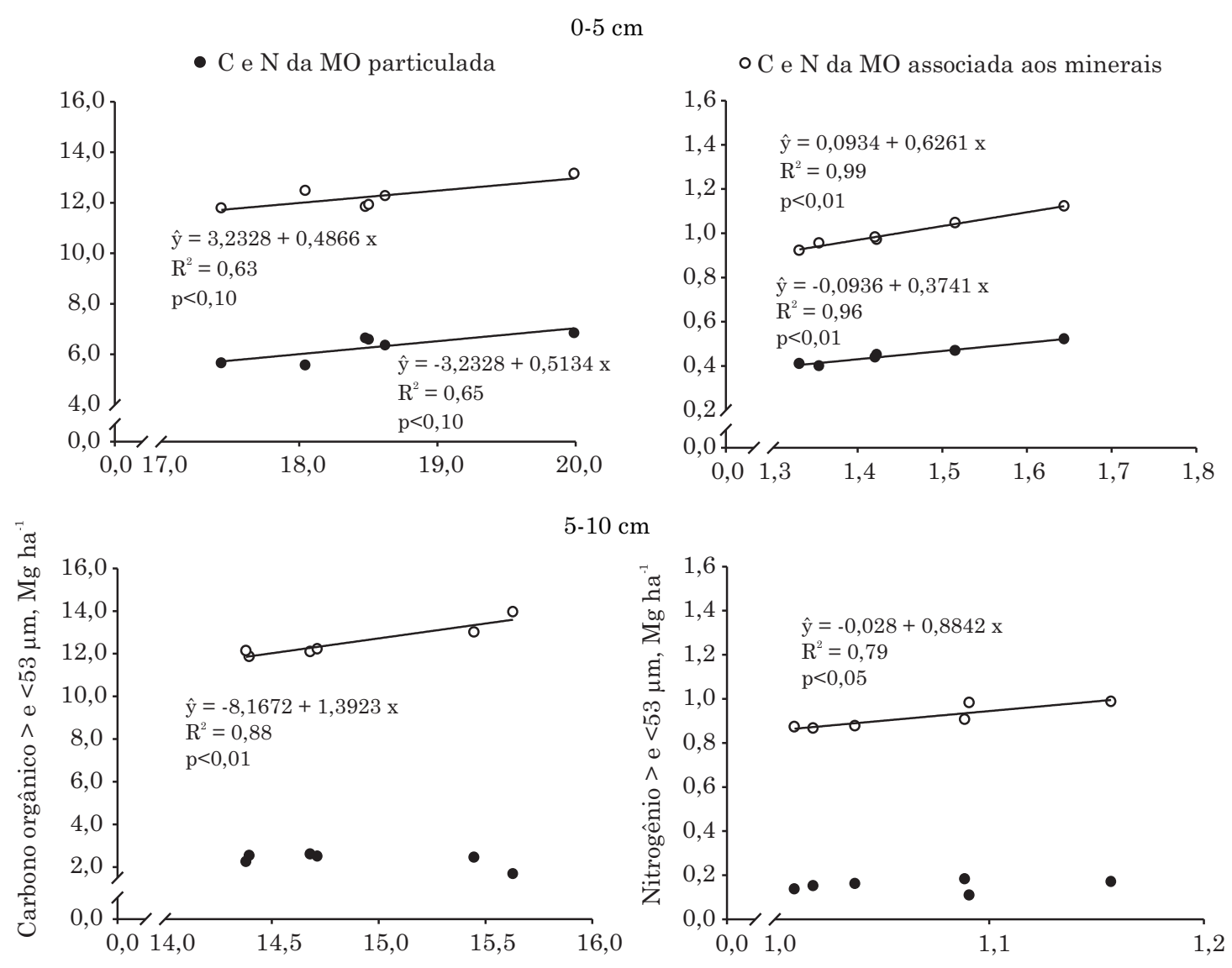

$10-20 \mathrm{~cm}$
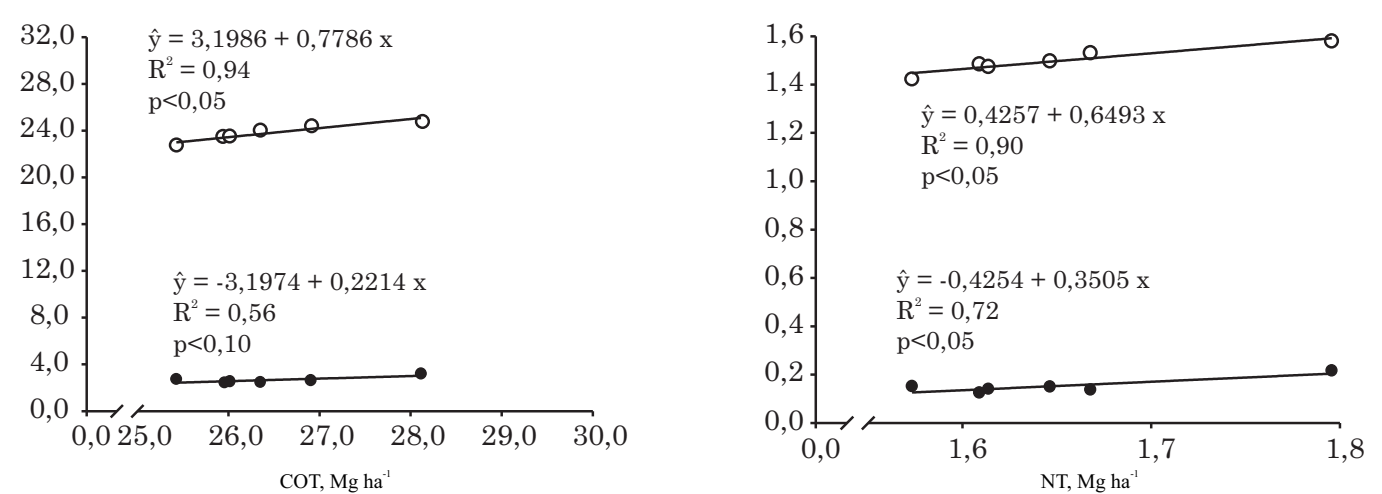

Figura 2. Regressão entre COT e NT com as frações granulométricas da matéria orgânica (MO) particulada $(>53 \mu \mathrm{m})$ e MO associada aos minerais $(<53 \mu \mathrm{m})$, nas camadas de 0-5, 5-10 e 10-20 cm, nos sistemas de culturas em rotação sob plantio direto há 21 anos, em Latossolo Vermelho distrófico típico. 
capacidade finita dos estoques de $\mathrm{C}$ e $\mathrm{N}$, em razão das características das ligações organominerais que ocorrem. Entretanto, na fração silte e areia, a MO associada a essas frações minerais é mais lábil, com interações mais fracas, o que diminui a sua proteção no solo. Neste estudo, como os estoques de $\mathrm{C}$ e $\mathrm{N}$ nas frações silte e argila foram avaliados conjuntamente (MO associada aos minerais), não foi possível perceber o comportamento reportado por Diekow et al. (2005). Observou-se um crescimento linear no estoque de $\mathrm{C} \mathrm{e}$ $\mathrm{N}$ nas frações silte + argila, onde possivelmente o estoque na fração silte interferiu fortemente nesses resultados, por apresentar um comportamento intermediário entre os padrões das frações argila e areia, ou seja, por conter porções de MO particulada e compostos orgânicos humificados (Balabane \& Plate, 2004; Diekow et al., 2005). Esse comportamento sugere que os sistemas avaliados ainda oferecem potencial em aumentar os estoques de $\mathrm{C} \mathrm{e} \mathrm{N}$, a partir de incrementos em ambas as frações, principalmente nos sistemas onde a entrada anual de $\mathrm{C}$ e $\mathrm{N}$ via parte aérea e sistema radicular é maior (Quadro 1). Portanto, os resultados indicam que, com o aumento da proporção de espécies na rotação com ciclo de vida mais longo e alto aporte de material orgânico ao solo, pode ocorrer aumento no estoque de MO em ambos os compartimentos avaliados e, consequentemente, favorecer a funcionalidade do solo, promovendo o incremento da sua qualidade.

\section{CONCLUSÕES}

1. Os estoques de $\mathrm{C}$ e $\mathrm{N}$ totais e a matéria orgânica particulada foram sensíveis em detectar as variações em sistemas de culturas em plantio direto na camada de $0-5 \mathrm{~cm}$, que por sua vez influenciou o estoque acumulado na de $0-20 \mathrm{~cm}$.

2. A adição de resíduos pelo sistema radicular e a sequência temporal das culturas foram determinantes para os estoques de $\mathrm{C}$ e $\mathrm{N}$ totais, nas frações granulométricas após 21 anos em plantio direto. Os maiores estoques ocorreram no sistema Alfafa-Milho em relação aos sistemas bianual Ervilhaca-MilhoTrigo-Soja e anual Trigo-Soja.

3. A matéria orgânica particulada contribuiu em torno de 30 \% para o carbono orgânico total na camada de $0-5 \mathrm{~cm}$, o que determinou sua sensibilidade em variar significativamente em razão do tipo de rotação de cultura estudado.

\section{LITERATURA CITADA}

ASHAGRIE, Y.; ZECH, W.; GUGGENBERGER, G. \& MAMO, T. Soil aggregation, and total and particulate organic matter following conversion of native forests to continuous cultivation in Ethiopia. Soil Till. Res., 94:101$108,2007$.
BALDOCK, J.A. \& SKJEMSTAD, J.O. Role of the soil matrix and minerals in protecting natural organic materials against biological attack. Org. Geochem., 31:697:710, 2000.

BALABANE, M. \& PLATE, A.F. Aggregation and carbon storage in silty soil using physical fractionation techniques. Eur. J. Soil. Sci., 55:415-427, 2004.

BALESDENT, J. \& BALABANE, M. Major contribution of roots to soil carbon storage inferred from maize cultivated soils. Soil Biol. Biochem., 28:1261-1263, 1996.

BALESDENT, J.; CHENU, C. \& BALABANE, M. Relationship of soil organic matter dynamics to physical protection and tillage. Soil Till. Res., 53:215-230, 2000.

BALOTA, E.L.; COLOZZI FILHO, A.; ANDRADE, D.S. \& DICK, R.P. Long-term tillage and crop rotation effects on microbial biomass and $\mathrm{C}$ and $\mathrm{N}$ mineralization in a Brazilian Oxisol. Soil Till. Res., 77:137-145, 2004.

BAYER, C.; MARTIN-NETO, L.; MIELNICZULK, J. \& PAVINATO, A. Armazenamento de carbono em frações lábeis da matéria orgânica de um Latossolo Vermelho sob plantio direto. Pesq. Agropec. Bras., 39:677-683, 2004.

BAYER, C.; MARTIN NETO, L.; MIELNICZUK, J.; PILLON, C.N. \& SANGOI, L. Changes in soil organic matter fractions under subtropical no-till cropping systems. Soil Sci. Soc. Am. J., 65:1473-1478, 2001.

BLAKE, G.R. \& HARTGE, K.H. Bulk density. In: KLUTE, A., ed. Methods of soil analysis. Physical and mineralogical methods. Madison, America Society of America, 1986. p.363-375.

CAMBARDELlA, C.C. \& ELLIOTT, E.T. Particulate soil organic-matter changes across a grassland cultivation sequence. Soil Sci. Soc. Am. J., 56:777-783, 1992.

CERRI, C.E.P.; SPAROVEK, G.; BERNOUX, M.; EASTERLING, W.E.; MELILLO, J.M. \& CERRI, C.C. Tropical agriculture and global warming: Impacts and mitigation options. Sci. Agric., 64:83-99, 2007.

CHEVAllier, T.; BLANCHART, E.; ALBRECHT, A. \& FELLER, C. The physical protection of soil organic carbon in aggregates: A mechanism of carbon storage in a Vertisol under pasture and market gardening (Martinique, West Indies). Agric. Ecosyst. Environ., 103:375-387, 2004.

CONCEIÇÃO, P.C.; AMADO, T.J.C.; MIELNICZUK, J. \& SPAGNOLLO, E. Qualidade do solo em sistemas de manejo avaliada pela dinâmica da matéria orgânica e atributos relacionados. R. Bras. Ci. Solo, 29:777-788, 2005 .

DIEKOW, J.; MIELNICZUK, J.; KNICKER, H.; BAYER, C.; DICK, D.P. \& KÖGEL-KNABNER, I. Carbon and nitrogen stocks in physical fractions of a subtropical Acrisol as influenced by long-term no-till cropping systems and $\mathrm{N}$ fertilization. Plant Soil, 268:319-328, 2005.

DUXBURY, J.J.; SMITH, M.S. \& DORAN, J.W. Soil organic matter as a source and a sink of plant nutrients. In: COLEMAN, D.C.; OADES, J.M. \& UEHARA, G., eds. Dynamics of soil organic matter in tropical ecosystems. Honolulu, University of Hawaii, 1989. p.33-67. 
EMPRESA BRASILEIRA DE PESQUISA AGROPECUÁRIA EMBRAPA. Centro Nacional de Pesquisa de Solos. Sistema brasileiro de classificação de solos. 2.ed. Rio de Janeiro, 2006. 306p.

FRANZLUEBBERS, A.J. Soil organic matter stratification ratio as an indicator of soil quality. Soil Till. Res., 66:95$106,2002$.

FREIRE, J.R.J. Fixação biológica de nitrogênio pela simbiose rizóbio/leguminosas. In: CARDOSO, E.J.B.N.; TSAI, S.M. \& NEVES, M.C.P., eds. Microbiologia do solo. Campinas, Sociedade Brasileira de Ciência do Solo, 1992. p.121155.

GREGORICH, E.G.; CARTER, M.R.; ANGERS, D.A.; MONREAL, C.M. \& ELLERT, B.H. Towards a minimum data set to assess soil organic matter quality in agricultural soils. Can. J. Soil Sci., 367-375, 1994.

INSTITUTO AGRONÔMICO DO PARANÁ - IAPAR. Disponível em: <http://www.iapar.br/arquivos/Image/monitoramento /Medias_Historicas/Ponta_Grossa.htm>. Acesso em: 24 out. 2011.

JANZEN, H.H.; CAMPBELL, C.A.; GREGORICH, E.G. \& ELLERT, B.H. Soil carbon dynamics in Canadian agroecosystems. In: LAL, R.; KIMBLE, J.M.; FOLLETT, R.F. \& STEWART, B.A., eds. Soil processes and the C cycle. Boca Raton, CRC Press, 1998. p.57-80.

KALBITZ, K. \& KAISER, K. Contribution of dissolved organic matter to carbon storage in forest mineral soils. J. Plant. Nutr. Soil. Sci., 171:52-60, 2008.

KUZYAKOV, Y.; FRIEDEL, J.K. \& STAHR, K. Review of mechanisms and quantification of priming effects. Soil Biol. Biochem., 32:1485-1498, 2000.

KUZYAKOV, Y. Review: Factors affecting rhizosphere priming effects. J. Plant. Nutr. Soil Sci., 165:382-396, 2002.

LOVATO, T.; MIELNICZUK, J.; BAYER, C. \& VEZZANI, F.M. Adição de carbono e nitrogênio e sua relação com os estoques no solo e com o rendimento do milho em sistemas de manejo. R. Bras. Ci. Solo, 28:175-187 2004.

OADES, J.M. An overview of processes affecting the cycling of organic carbon in soils. In: ZEPP, R.G. \& SONNTAG, C., eds. Role of non-living organic matter in the earth's carbon cycle. New York, John Wiley, 1995. p.55-94.

RASSE, D.P.; RUMPEL, C. \& DIGNAC, M.O. Is soil carbon mostly root carbon? Mechanisms for a speciûc stabilization. Plant Soil, 269:341-356, 2005.
SANTOS, N.Z.; DIECKOW, J.; BAYER, C.;MOLIN, R.; FAVARETTO, N.; PAULETTI, V. \& PIVA, J.T. Forages, cover crops and related shoot and root additions in no-till rotations to $\mathrm{C}$ sequestration in a subtropical Ferralsol. Soil Till. Res., 111:208-218, 2011.

SISTI, C.P.J.; SANTOS, H.P.; KOHHANN, R.; ALVES, B.J.R.; URQUIAGA, S. \& BODDEY, R.M. Change in carbon and nitrogen stocks in soil under 13 years of conventional or zero tillage in southern Brazil. Soil Till. Res., 76:39-58, 2004.

SIX, J.; ELLIOTT, E.T. \& PAUSTIAN, K. Soil macroaggregate turnover and microaggregate formation: a mechanism for $\mathrm{C}$ sequestration under no-tillage agriculture. Soil Biol. Biochem., 32:2099-2103, 2000.

SIX, J.; FELLER, C.; DENEF, K.; OGLE, S.M. \& SA, M.J.C. Soil organic matter, biota and aggregation in temperate and tropical soils: effects of no-tillage. Agronomie, 22:755$775,2002 \mathrm{a}$.

SIX, J.; CONANT, R.T.; PAUL, E.A. \& PAUSTIAN, K. Stabilization mechanisms of soil organic matter: Implications for Csaturation of soils. Plant Soil, 241:155-176, 2002b.

SIX, J.; BOSSUYT, H.; DEGRYZE, S. \& DENEF, K. A history of research on the link between (micro)aggregates, soil biota, and soil organic matter dynamics. Soil Till. Res., 79:7-31, 2004.

SHUKLA, M.K.; LAL, R. \& EBINGER, M. Determining soil quality indicators by factor analysis. Soil Till. Res., 87:194204, 2006.

VEZZANI, F.M. \& MIELNICZUK, J. Agregação e estoque de carbono em Argissolo submetido a diferentes práticas de manejo agrícola. R. Bras. Ci. Solo, 35:213-223, 2011.

VIEIRA, F.C.B.; BAYER, C.; ZANATTA, J.A.; MIELNICZUK, J. \& SIX, J. Building up organic matter in a subtropical Paleudult under legume cover-crop-based rotations. Soil Sci. Soc. Am. J., 73:1699-1706, 2009.

WEBER, M.A. \& MIELNICZUK, J. Estoque e disponibilidade de nitrogênio no solo em experimento de longa duração. R. Bras. Ci. Solo, 33:429-437, 2009.

ZANATTA, J.A.; BAYER, C.; DIECKOW, J.; VIEIRA, F.C.B. \& MIELNICZUK, J. Soil organic carbon accumulation and carbon costs related to tillage, cropping systems and nitrogen fertilization in a subtropical Acrisol. Soil Till. Res., 94:510-519, 2007.

ZHU, Y.P.; SHEAFFER, C.C.; VANCE, C.P.; GRAHAM, P.H.; RUSSELLE, M.P. \& MONTEALEGRE, C.M. Inoculation and nitrogen affect herbage and symbiotic properties of annual Medicago species. Agron. J., 90:781-786, 1998. 